\title{
The impact of migration on tuberculosis epidemiology and control in high-income countries: a review
}

\author{
Manish Pareek ${ }^{1,2^{*}}$ (D), Christina Greenaway ${ }^{3}$, Teymur Noori ${ }^{4}$, Jose Munoz ${ }^{5}$ and Dominik Zenner ${ }^{6,7}$
}

\begin{abstract}
Tuberculosis (TB) causes significant morbidity and mortality in high-income countries with foreign-born individuals bearing a disproportionate burden of the overall TB case burden in these countries. In this review of tuberculosis and migration we discuss the impact of migration on the epidemiology of TB in low burden countries, describe the various screening strategies to address this issue, review the yield and cost-effectiveness of these programs and describe the gaps in knowledge as well as possible future solutions.

The reasons for the TB burden in the migrant population are likely to be the reactivation of remotely-acquired latent tuberculosis infection (LTBI) following migration from low/intermediate-income high TB burden settings to high-income, low TB burden countries.

TB control in high-income countries has historically focused on the early identification and treatment of active TB with accompanying contact-tracing. In the face of the TB case-load in migrant populations, however, there is ongoing discussion about how best to identify TB in migrant populations. In general, countries have generally focused on two methods: identification of active TB (either at/post-arrival or increasingly pre-arrival in countries of origin) and secondly, conditionally supported by WHO guidance, through identifying LTBI in migrants from high TB burden countries. Although health-economic analyses have shown that TB control in high income settings would benefit from providing targeted LTBI screening and treatment to certain migrants from high TB burden countries, implementation issues and barriers such as sub-optimal treatment completion will need to be addressed to ensure program efficacy.
\end{abstract}

Keywords: Migration, Tuberculosis, Screening, Review

\section{Background}

In this review (see Table 1) we first analyse the burden of tuberculosis (TB) in foreign-born, migrant populations before going on to discuss the drivers of the current TB epidemiology in these populations focusing on migration patterns, the importance of reactivation of latent tuberculosis infection as compared to the burden of imported active TB and molecular genotyping data underpinning these studies. We then go on discuss, in detail, the methods, outcomes, and cost-effectiveness of

\footnotetext{
*Correspondence: mp426@le.ac.uk

'Department of Infection, Immunity and Inflammation, University of Leicester, Leicester, UK

2Department of Infection and HIV Medicine, University Hospitals of Leicester NHS Trust, Leicester, UK

Full list of author information is available at the end of the article
}

the different TB control strategies in place for migrant populations.

\section{Tuberculosis epidemiology in high-income countries}

Tuberculosis (TB) remains a 'global health emergency' [1]. Although much of the burden is concentrated in high-burden settings in Asia and Africa (which make up $58 \%$ and $28 \%$ of all cases respectively) [2], TB continues to be of concern in high-income nations. In the 34 highincome Organisation for Economic Cooperation and Development (OECD) countries, TB incidence fell by a median of $4.7 \%$ per year (between 1995 and 2004) decelerating to $3.0 \%$ per year between 2005 and 2014 [3] making TB elimination more difficult to attain [4]. 
Table 1 Key messages about tuberculosis and migration in high-income countries

- Tuberculosis continues to be a public health concern in high-income countries

- Tuberculosis burden in high-income countries is primarily amongst the foreign-born, migrant population

- The reasons underlying this burden are the interaction of migration from high TB burden countries and the reactivation of remotely acquire latent tuberculosis infection in the first five years after arrival

- Genotyping data suggests that there is relatively little transmission in migrant communities in the receiving country

- Methods of TB control in migrant population have historically focused on identifying active tuberculosis but the yields for this remain relatively low

- Screening migrants for latent tuberculosis infection may have a higher yield although implementation may be difficult

- The health economics of screening migrants for active and/or latent tuberculosis is a topic of much debate

- Targeted pre-arrival screening for active TB and post arrival screening for latent tuberculosis infection in migrants from intermediate/high TB burden settings may provide the most cost-effective solution

- Implementation of programmatic screening is limited by uptake, acceptance and completion of therapy

Yet the overall changes seen in TB incidence in highincome countries hide an important disparity: while local-born cases have remained static or decreased, foreign-born cases have decreased more slowly or increased. From 2000 to 2013, local-born TB cases decreased by half (median $51.3 \%$; IQR $-64.3--35.3 \%$ ) whilst foreign-born case notifications increased marginally (median $2.3 \%$; IQR $-36.7-+40.4 \%$ ) [5-10]. In just under half of the high-income OECD countries foreignborn TB cases increased [5-10]. Consequently foreignborn individuals, in 2013, made up over half of all TB cases (median 52.0 \%; IQR 31.4-73.9 \%; Fig. 1) [5-11] with incidence rates 8.7-18.4 times that seen in the local-born population $[7,9,10,12]$.

Drilling down further into the patterns TB notifications in the foreign-born population in high-income countries reveals information about key risk groups with the highest incidence and risk of active $\mathrm{TB}$ following migration: migrants from Asia and Africa where the burden of TB is moderate/high, recent migrants (within 5 years of arrival), refugees and individuals with comorbidities (such as HIV infection and diabetes mellitus) $[7,9,10,12,13]$.

\section{Migration and reactivation of latent TB infection: key drivers of tuberculosis in migrants in high- income countries}

Understanding the scale and nature of migration to high-income countries

In 2013, United Nations figures showed that the global number of migrants was 232 million - a $50 \%$ increase over the preceding two decades [14] with a concomitant change in the pattern of sending countries [15] as globalisation, conflict, and financial reasons have become increasingly important drivers of migration flows. This has resulted in more permanent migrants moving from low/medium income, higher TB burden, countries to high-income developed, lower TB burden, countries including USA, Canada, Australia and Western European nations although migrants are not necessarily representative of the population in the country of origin [15]. Top migrant sending countries for each high-income country will vary according to historic, linguistic, cultural links and geographic proximity. In the UK a significant proportion of the foreign-born migrants arrive from former colonies in Sub-Saharan Africa and the Indian Subcontinent whereas in the US the majority of the foreign-born population originate from Central and South America [16]. As a consequence of migration, in high-income OECD countries, the median proportion of the population that is foreign-born is estimated to be 13.7 \% (IQR 10.8-18.4 \%) [17].

\section{Reactivation of latent TB infection in determining TB burden in migrants}

The TB burden observed in foreign-born individuals occurs due to one of three reasons: (1) migrants from overseas must either have active TB on arrival, (2) migrants have remotely-acquired latent TB infection which reactivates post-arrival or (3) migrants acquire TB, following arrival, through local transmission (Fig. 2).

\section{Active TB disease in migrants on arrival in the receiving country}

Surveillance data, and findings from previous metaanalyses (Table 2), have shown that the proportion of migrants with active TB present at the time of migration is relatively small $(0.35 \%)[18,19]$.

\section{High prevalence of latent $T B$ infection and risk of progression to active disease}

Latent TB prevalence figures in migrants are primarily derived from cross-sectional studies where, depending on the specific population tested and the diagnostic tool used, $5-72 \%$ of migrants test positive for LTBI [20-43]; this is independently associated with increasing age and TB incidence in country-of-origin [41, 42, 44]. One can therefore infer that it is both the cumulative duration of exposure and the TB burden in the source country which determines whether individuals will have LTBI [29, 41, 42, 44, 45]. Migrants with LTBI are coming to lower incidence settings and in the initial years following arrival in the destination country, have a higher risk of LTBI reactivation which decreases slowly over time but remains higher than rates in the 


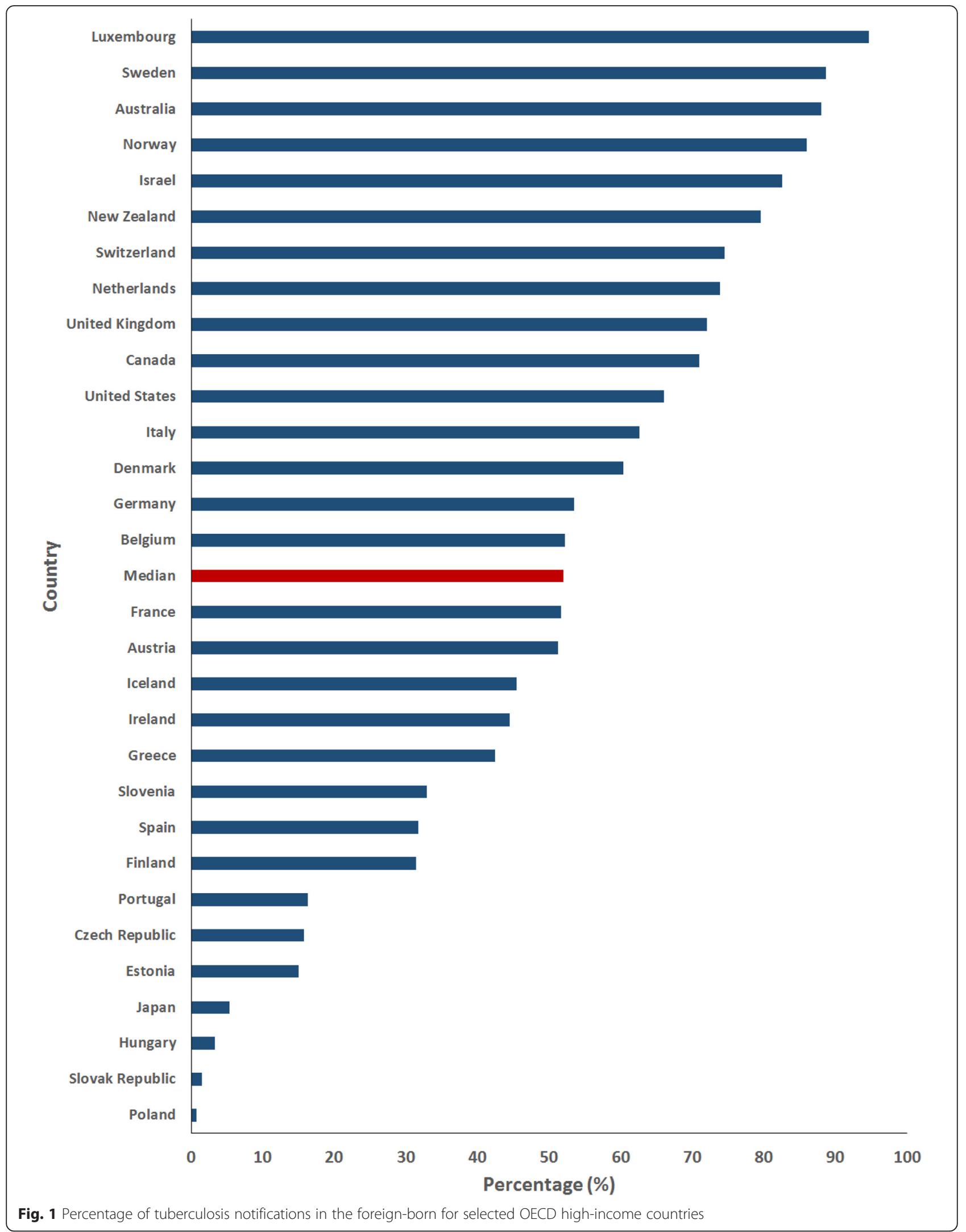




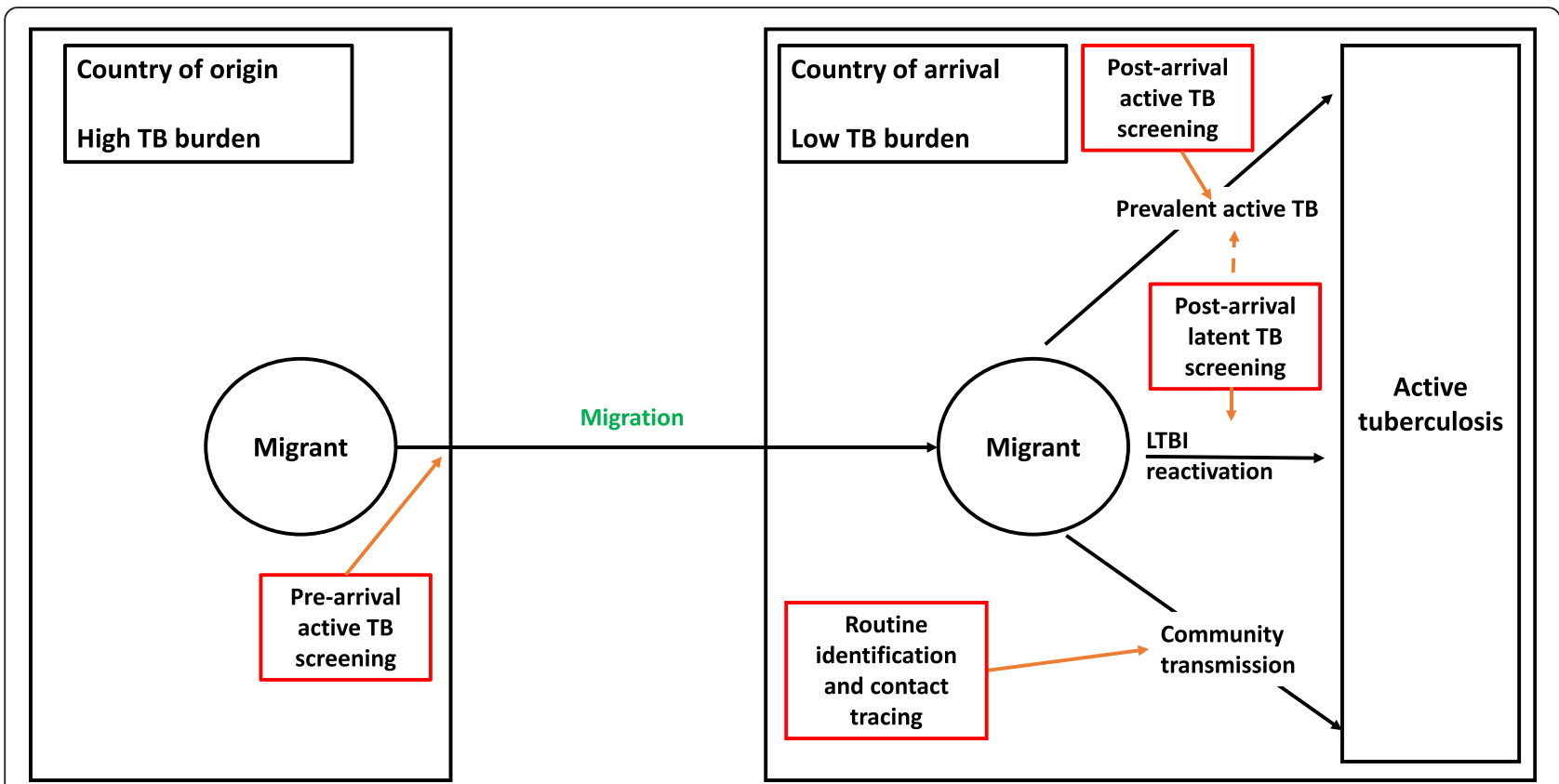

Fig. 2 Schematic diagram of migration, factors determining how incident active tuberculosis occurs and methods of screening migrants. Footnote: As a by-product of post-arrival latent TB screening, some cases of prevalent active TB may be identified

host population $[12,46-48]$ This higher rate of reactivation in the initial one to two years after migrants arrive likely reflects latent tuberculosis infection which has been acquired in their country of origin shortly before migration although there is also likely to be an ongoing complex interplay, in the destination country, of host (such as age, and comorbidities including diabetes mellitus) and environmental factors (such as nutritional status) which contribute to the observed epidemiology. Understanding the natural history of TB in recently arrived migrants is important when we are considering how best to implement TB control in this population.

Whilst the literature has expanded rapidly in respect of cross-sectional data on LTBI prevalence, longitudinal data on the risk of migrants with diagnosed, untreated, LTBI progressing to active TB disease remain limited partially due to low numbers of chemoprophylaxis-naive patients (due to recommendations to treat, and not

Table 2 Yields for active tuberculosis from previous meta-analyses

\begin{tabular}{lccll}
\hline Author & Year & \multicolumn{3}{c}{ Yield for active tuberculosis (\%) } \\
\cline { 3 - 5 } & & Overall & Pre-arrival & At/post-arrival \\
\hline Klinkenberg [19] & 2009 & 0.35 & 1.21 & 0.31 \\
& & 0.51 & & \\
Arshad [18] & 2010 & 0.35 & - & 0.35 \\
Aldridge [71] & 2014 & 0.22 & 0.22 & - \\
\hline
\end{tabular}

withhold treatment from, individuals identified with latent TB such as in the UK) and because studies to answer this research question need a large sample size and long duration of follow-up. A UK study followed migrants, predominantly from the Indian Subcontinent, and found a TB progression rate of $16.3 \%$ amongst untreated tuberculin skin test (TST) positive patients over a 15 year period following UK arrival [49]. The risk was significantly higher for young migrants (aged 16-19) and for women [49]. However this study did not adjust incidence rates for travel back to countries of origin nor underlying medical comorbidities. A Norwegian study of asylum seekers found a progression rate of $1.1 \%$ over a follow-up period of up to 32 months [50]. By contrast, Marks and colleagues evaluated a large cohort of predominantly Southeast Asian migrants, with no evidence of active TB, over a mean follow-up of 10.3 years postarrival in Australia and found that $0.12 \%$ with a positive TST progressed to active TB disease per year [51]. Further work is needed to determine both the overall risk of progression from LTBI to active TB disease in migrants as well as the contribution of concomitant medical comorbidities and demographics such as diabetes mellitus, chronic kidney disease and age. Diabetes mellitus and chronic kidney disease are more common in migrant populations and significantly increase the risk of reactivation from LTBI to active $\mathrm{TB}[13,52]$. This is likely to result in increasing $\mathrm{TB}$ notifications in the foreign-born, migrant populations, particularly as the migrant population ages, and this will, therefore, need 
to be taken into consideration when developing $\mathrm{TB}$ control programmes [13].

An additional issue which is likely to play a part in determining $\mathrm{TB}$ epidemiology in migrant populations is the acquisition, and subsequent reactivation, of LTBI following re-exposure during travel back to their countries of origin. Previous work in this area has indicated that travel to high TB burden countries increases the risk of acquiring LTBI with the risk increasing with more prolonged travel and a higher TB burden in the country visited [53]. Although there is a paucity of data on the proportion of TB acquired through travel, published work suggests this could be anywhere between $20 \%$ and $50 \%[54,55]$. However further prospective research in this area is needed to more accurately quantify the risk.

\section{Using molecular genotyping to distinguish reactivation of latent $T B$ and recent transmission of active TB in migrants}

In order to distinguish reactivation of remotely acquired LTBI from TB transmission, authors have genotyped DNA isolated from $M$. tuberculosis cases in foreign-born individuals. Here, individuals with a TB isolate with a clustered DNA pattern that matches at least one other case in the cohort is attributed to recent transmission whereas cases with unique, DNA patterns are thought to arise due to the reactivation of LTBI $[56,57]$. In a large meta-analysis across a range of $\mathrm{TB}$ burden settings, foreign-born individuals were significantly less likely to have a clustered isolate as compared to local-born individuals ( $25 \%$ versus $45.8 \%$ clustered respectively) [57]. Moreover, there is little evidence that the foreign-born population transmit $\mathrm{TB}$ to the local born population [58]. These data, in conjunction with TB surveillance data, appear to highlight the importance of reactivating LTBI amongst migrants in determining TB epidemiology in high-income countries and, therefore, the need to have appropriate control measures in place.

\section{Tuberculosis control with a special focus on migrants}

Globally, TB control policies focus on quickly diagnosing and treating individuals with active TB. Additionally in high-income countries this is complemented by the contact-tracing of household contacts of smear-positive cases with the overall aim of reducing onward transmission. However this method of TB control does not fully address the potential source of reactivation of remotely acquired LTBI progressing to active TB disease - such as that seen in migrants. Dynamic transmission models have been used to study the impact of chemoprophylaxis for LTBI on global TB control and concluded that concurrently targeting individuals with active TB disease and individuals with LTBI will augment TB control [59-63]. The growing importance of tackling LTBI is reflected by recent WHO guidelines which conditionally recommend migrants from high TB burden countries are offered screening and LTBI treatment [64].

\section{Migrant screening practices and their outcomes in high-income countries}

Several authors have recently reviewed the migrant TB screening programmes in high-income countries. Each program is different and they differ by whether screening is done for active or latent TB (or both), when screening is performed in relation to arrival in the host country, which groups of migrants are screened (refugees or other migrants groups; which countries of origin) and which tools are used to screen for active and latent TB (see Table 3) [65-69]. It should, however, be borne in mind that much of the available data relates to documented migrants and there remains ongoing difficulty in collecting data on undocumented migrant who bypass standard screening protocols.

\section{Screening practices for active tuberculosis}

Most OECD high-income countries screen migrants for active TB although the specifics of how this is performed vary significantly across countries. Most active TB screening programs in Western Europe are performed on or soon after arrival with a chest radiograph (CXR). Other countries such as Canada, the US, Australia, New Zealand and recently the UK, screen for active TB with a CXR prior to arrival. If the CXR is abnormal a sputum smear and culture are performed. Those found to have active TB are treated prior to arrival and granted permission to enter the country if cultures are negative at the end of treatment. Those found to have an abnormal CXR but negative sputum cultures or those with prior treated TB are followed after arrival in a post-landing surveillance program [65-70].

The criteria for which migrant groups are screened is also highly variable [67, 69]. Alvarez and colleagues found that countries differed in which migrants were selected for screening basing their decision on a number of factors including: type of migrant (e.g. refugee, students, workers), duration of stay, intended occupation or TB burden in country of origin [67]. In a large survey of OECD countries, the authors identified heterogeneity in source country incidence thresholds for screening although, in general, migrants arriving from high TB burden settings were preferentially selected for screening [69]. The reasons for this remain unclear but it may reflect a lack of evidence in this area.

\section{Outcomes of screening for active $T B$}

Whilst screening for active TB is frequently undertaken, there is a lack of trial data for its effectiveness as a public health intervention. Policy decisions therefore rely on 
Table 3 Potential strengths and weaknesses of different migrant screening methods

\begin{tabular}{lll}
\hline & Screening methodology & \\
\cline { 2 - 3 } Screening tool used & Chest x-ray & Screening for latent tuberculosis infection \\
Screening location & Pre-arrival & $\begin{array}{l}\text { Tuberculin skin test } \\
\text { Interferon gamma release assay }\end{array}$ \\
& At arrival & Post-arrival \\
Post-arrival & \\
Strengths & Able to identify active TB & \\
& Able to identify infectious individuals & Identifies latent TB before reactivation occurs \\
& Can be integrated into immigration processes & Can be built into community programmes \\
Weaknesses & Low yields for active TB & Targeted screening likely to be cost-effective \\
& Uncertain cost-effectiveness (unless screening targeted) & Programmatically difficult to implement \\
& Does not identify patients with latent TB who can go & Numbers accepting and completing treatment may \\
& on to reactivate & be suboptimal
\end{tabular}

national evaluations and cross-sectional observational data.

Pre-arrival screening, in country of origin, is part of the immigration process of several high-income countries including the US, Australia, Canada and the UK. Aldridge and colleagues undertook a detailed systematic review and meta-analysis on the yields of active TB through prearrival screening. They found that the overall yield for culture positive active TB was $0.22 \%$ (219 cases/100,000) [71]; the yield for culture positive TB increased with increasing TB prevalence in country of origin [71] suggesting that setting an incidence threshold for pre-arrival screening of migrants may be needed to ensure costeffective use of resources. Klinkenberg et al. reviewed the yields for active TB at different stages of the immigration process and found that the yields for pre-arrival, as compared to post-arrival, screening were higher $(1.21 \%$ versus $0.31 \%$ respectively) although these data were based entirely on non-EU studies [19]. A recently published analysis of the US pre-arrival screening programme found that 4032 cases of culture positive TB were diagnosed amongst 1,561,460 migrants screened (yield $0.26 \%$ ) resulting in a reduction in the number of $\mathrm{TB}$ cases diagnosed amongst migrants within one year of arrival in the US [43]. The UK pre-arrival screening programme yield for active TB has steadily increased from $0.05 \%$ in 2006 to $0.16 \%$ in 2014 which most likely reflects the use of sputum cultures [72, 73].

Post-arrival screening for active TB involves chest radiography once the migrant has arrived in the host country. Two systematic reviews have evaluated the outcomes and yields for active TB in high-income countries $[18,19]$. Arshad et al. reviewed 22 studies comprising $2,620,739$ migrants and found that the overall yield for post-arrival screening was $0.35 \%$ with higher yields in migrants from Africa and Asia [18]. Klinkenberg and colleagues reviewed 40 studies and found that the yields for post-arrival screening, which were lower than that for pre-arrival screening, ranged from $0.20 \%$ to $0.36 \%$ depending on the specific setting in which screening was undertaken [19].

Previous work has shown that the current models of active TB screening have weaknesses including: individuals not completing the screening processes, limited yields for active disease and an inability to identify active TB occurring through LTBI reactivation [13].

\section{Screening practices for latent tuberculosis}

Whilst most countries offer some form of screening for active TB, screening for LTBI is much less commonly performed [69]. High-income countries that screen for LTBI usually undertake this post-arrival with a tuberculin skin test (TST) or an interferon gamma release assay (IGRA) [69, 74].

For reasons of practicality and cost-effectiveness, most high-income countries attempt to limit the eligible population to refugees or asylum seekers or those individuals arriving from high TB burden settings [69]. However, countries vary considerably in their definition of a high TB burden setting for the purposes of migrant screening [69]; the UK has taken a decision to screen migrants arriving from countries in Sub-Saharan Africa or those countries with a TB incidence above 150 per 100,000 whereas Canada screens at a lower threshold of $30 / 100,000$ but only migrants with increased risk of reactivation. Given the prevalence of comorbidities in the migrant population (such as diabetes mellitus,) which increase the risk of reactivation, these factors will likely need to be taken into account when determining which migrants to screen. At the present time, however, the 
variation between guidelines could reflect uncertainty about the optimal screening threshold which balances the need to identify the majority of LTBI with cost effectiveness.

\section{Outcomes of screening for latent tuberculosis infection}

Successful screening for LTBI involves a number of key interlinked steps including the accurate identification of migrants, appropriate screening of migrants, initiation of chemoprophylaxis and completion of therapy. However most of the data on LTBI screening outcomes come from cross-sectional studies which have been conducted with the primary aim of calculating the prevalence of LTBI in migrants (estimated at around $25-30 \%$ in young adult migrants from high incidence countries) [20-43]. However, there is less data on the other elements of the screening pathway - including uptake, and completion, of chemoprophylaxis. A Canadian group recently reviewed the data on LTBI screening effectiveness and found that migrants dropped out at each step of the screening pathway so that overall only $31 \%$ of the cohort completed the programme successfully highlighting the need for research into interventions to optimise the LTBI screening pathway [75]. Programmatically, at a national level, there is little observational data on the impact of LTBI screening on TB notifications in migrants although the recently commenced UK national migrant screening programme will provide this useful data in the next few years [76].

\section{Health economics of migrant screening}

Whilst the programmatic outcomes of migrant screening are important, a key consideration for policy-makers and clinicians is cost-effectiveness. Several studies have explored the cost-effectiveness of migrant screening for TB although they have focused on different aspects of screening including whether to screen for active or latent $\mathrm{TB}$, which migrant groups to screen and how to screen $[41,42,77-86]$.

\section{Cost-effectiveness of screening for active and latent tuberculosis}

Screening migrants for active TB is widely implemented by high-income countries albeit with different models of care. However there are few studies formally examining the cost-effectiveness of this intervention. Previous studies have come to differing conclusions about the costeffectiveness of screening migrants for active TB. Schwartzman and colleagues modelled the comparative cost-effectiveness of migrant screening using chest radiography versus tuberculin skin test (versus no screening) and found that in migrants with a high prevalence of infection that chest radiography was the most costeffective screening modality although the TST strategy prevented the most cases of TB. By contrast, Dasgupta et al. constructed a Markov model informed by empirical data to compare the cost effectiveness of screening migrants with chest radiography pre-arrival followed by TST (if the CXR showed any abnormalities) with screening close contacts of index sputum smear-positive cases (with TST followed by CXR) [77]. The authors found that migrant screening using chest radiography was not cost-effective due to difficulties with operationalising screening [77]. The lack of research in this area highlights the need for further health-economic analyses to objectively assess, and make conclusions about, the costeffectiveness of screening migrants for active TB.

A larger number of published studies from highincome countries have focused on evaluating the costeffectiveness of screening migrants for LTBI [78-86]. These studies, which have evaluated different aspects of LTBI screening including which migrants to screen and how to screen, have generally concluded that LTBI screening of migrants from high burden countries, mainly Asia and Africa is a cost-effective intervention in high-income countries [41, 42, 78-86].

Methods for diagnosing LTBI have evolved over the last decade with IGRAs increasingly replacing the TST [87]. This is reflected by the several studies which have explored the relative cost-effectiveness of different screening modalities and algorithms for LTBI [41, 42, 79-84]. These health-economic analyses have, in general, found that IGRA are more cost-effective than TST $[41,42,79,81,84]$. However, in the absence of robust longitudinal data as for TST, there remains ongoing debate about the use of IGRA as a screening tool with certain national guidelines instead advocating the use of TST [13].

\section{Limitations of cost-effectiveness studies}

The scarcity of randomised clinical trials has meant that policy decision are mostly based on of health-economic modelling and observational data. However models must, by definition, make a number of simplifying assumptions and their results are therefore highly dependent on model structure and specific model parameters - even if there is uncertainty around these due to a lack of empirical data.

\section{Future directions}

The impact of migration on tuberculosis epidemiology in high-income countries is increasingly well-recognised and there has been a shift towards augmenting TB control by screening migrants for TB. Up until recently there has been a lack of coordinated international guidance in this area but the European Centre for Disease Prevention and Control (ECDC) is currently formulating guidance on the screening of migrants for a range of infectious diseases - including TB [88]. As migrant 
screening for TB becomes more embedded into TB control programmes, there will be an increasing need for high-quality operational research to establish how to undertake TB screening most effectively and integrate it with migrant health programmes including testing for blood-borne viruses.

\section{Conclusions}

In this review we have comprehensively brought together the literature with respect to all aspects of tuberculosis and migration. Tuberculosis in high-income countries continues to be a cause of morbidity and mortality - particularly amongst individuals who have been born overseas in high TB burden, low-income countries and migrated to high-income countries. The reasons for the burden of disease in the foreign-born, migrant, population are primarily due to migration from high $\mathrm{TB}$ burden settings and the reactivation of remotely-acquired latent TB infection. As a consequence there is increasing focus on how best to enhance TB control through the coordinated screening of migrants for TB. Whilst most countries focus on screening migrants for active $\mathrm{TB}$, this has a relatively low yield on its own and it is likely that the most effective and costeffective means of screening migrants for TB will comprise multiple, inter-linking elements: pre-arrival screening for active TB and targeted post arrival screening for LTBI in migrants from intermediate/high TB burden settings. However, the programmatic implementation of migrant screening is potentially hampered by limited uptake, acceptance and completion of therapy. There is an urgent need for further coordinated research in this area to inform future national and international guidance.

\section{Ethical approval}

Ethical approval not required as this is a literature review.

\section{Competing interest}

DZ is head of the tuberculosis screening unit at Public Health England and has responsibilities for quality assurance within the UK pre-entry screening programme and leads on the UK LTBI screening programme; MP, DZ, CG, TN and JM declare that they have no other conflict of interest.

\section{Authors' contributions}

MP conceived of the idea for the review. MP wrote the first draft of the manuscript with subsequent revisions made by all other co-authors. All co-authors had sight of the submitted paper. MP is guarantor for the paper. All authors read and approved the final manuscript.

\section{Funding}

This report is independent research supported by the National Institute for Health Research (NIHR Post-Doctoral Fellowship, Dr Manish Pareek, PDF-2015-08-102). The views expressed in this publication are those of the author(s) and not necessarily those of the NHS, the National Institute for Health Research or the Department of Health.

\section{Author details}

'Department of Infection, Immunity and Inflammation, University of Leicester, Leicester, UK. ${ }^{2}$ Department of Infection and HIV Medicine, University Hospitals of Leicester NHS Trust, Leicester, UK. ${ }^{3}$ Division of Infectious Diseases and Department of Clinical Epidemiology, Jewish General Hospital, McGill University, Montreal, Canada. ${ }^{4}$ European Centre for Disease Prevention and Control, Solna, Sweden. ${ }^{5}$ Barcelona Institute for Global Health, Barcelona, Spain. ${ }^{6}$ Centre for Infectious Disease Surveillance and Control, Public Health England, London, UK. ${ }^{7}$ Centre for Infectious Disease Epidemiology, University College London, London, UK.

Received: 2 February 2016 Accepted: 8 March 2016

Published online: 23 March 2016

\section{References}

1. World Health Organisation. WHO declares tuberculosis a global emergency. Soz Praventivmed. 1993;38:251-2.

2. World Health Organisation. Global tuberculosis report. Geneva: WHO; 2015.

3. Global Health Observatory Database. World Health Organisation, 2015. At http://apps.who.int/gho/data/?theme=main. Accessed 18 Dec 2015.

4. World Health Organisation Stop TB Partnership. The global plan to stop TB 2011-2015. Geneva: World Health Organisation; 2010.

5. EURO-TB. EuroTB and the national coordinators for tuberculosis surveillance in the WHO European Region. Surveillance of tuberculosis in Europe. Report on tuberculosis cases notified in 2000. Saint-Maurice: European Commission; 2000

6. European Centre for Disease Prevention and Control/WHO Regional Office for Europe. Tuberculosis surveillance in Europe 2013. Stockholm: European Centre for Disease Prevention and Control; 2015.

7. CDC. Reported tuberculosis in the United States. Atlanta: Department of Health and Human Services, CDC; 2015.

8. Japan Research Institute of Tuberculosis. Tuberculosis annual report 2013: TB in foreigners. Tokyo: JATA; 2013.

9. Public Health Agency of Canada. Tuberculosis in Canada 2013. Ottawa: Public Health Agency of Canada; 2015.

10. Toms $C$, Stapledon R, Waring J, Douglas P. Tuberculosis notifications in Australia 2012 and 2013. Commun Dis Intell. 2015;39:E217-35.

11. Institute of Environmental Science and Research Ltd (ESR). Tuberculosis in New Zealand: Annual Report 2013. Porirua: ESR; 2015.

12. Public Health England. Tuberculosis in the UK: 2014 report. London: Public Health England; 2014.

13. Greenaway C, Khan K, Schwartzman K. Tuberculosis surveillance and screening in selected high-risk populations. Ottawa: Public Health Agency of Canada; 2014.

14. United Nations Department of Economic and Social Affairs Population Division. International migration report 2013. Geneva: United Nations; 2013.

15. International Organisation for Migration. World migration report 2015. Geneva: IOM; 2015.

16. Gilbert RL, Antoine D, French CE, Abubakar I, Watson JM, Jones JA. The impact of immigration on tuberculosis rates in the United Kingdom compared with other European countries. Int J Tuberc Lung Dis. 2009;13:645-51.

17. Foreign-born population (indicator). 2016. At https://data.oecd.org/ migration/foreign-born-population.htm\#indicator-chart. Accessed 19 Jan 2016.

18. Arshad S, Bavan L, Gajari K, Paget SNJ, Baussano I. Active screening at entry for tuberculosis among new immigrants: a systematic review and metaanalysis. Eur Respir J. 2010;35:1336-45.

19. Klinkenberg E, Manissero D, Semenza JC, Verver S. Migrant tuberculosis screening in the EU/EEA: yield, coverage and limitations. Eur Respir J. 2009;34:1180-9.

20. Bothamley GH, Rowan JP, Griffiths CJ, et al. Screening for tuberculosis: the port of arrival scheme compared with screening in general practice and the homeless. Thorax. 2002;57:45-9.

21. Carvalho AC, Pezzoli MC, El-Hamad I, et al. QuantiFERON-TB Gold test in the identification of latent tuberculosis infection in immigrants. J Infect. 2007;55:164-8.

22. Carvalho AC, Saleri N, El-Hamad I, et al. Completion of screening for latent tuberculosis infection among immigrants. Epidemiol Infect. 2005;133:179-85. 
23. Gany FM, Trinh-Shevrin C, Changrani J. Drive-by readings: a creative strategy for tuberculosis control among immigrants. Am J Public Health. 2005;95:117-9.

24. Gibney KB, Mihrshahi S, Torresi J, Marshall C, Leder K, Biggs B-A. The profile of health problems in African immigrants attending an infectious disease unit in Melbourne, Australia. Am J Trop Med Hyg. 2009;80:805-11.

25. Haley CA, Cain KP, Yu C, Garman KF, Wells CD, Laserson KF. Risk-based screening for latent tuberculosis infection. South Med J. 2008;101:142-9.

26. Hernandez-Garduno E, Elwood RK. The prevalence and predictors of tuberculin positivity in subjects screened for reasons other than contact investigation. Can Respir J. 2008;15:181-7.

27. Janssens JP, Roux-Lombard P, Perneger T, Metzger M, Vivien R, Rochat T. Contribution of a IFN-gamma assay in contact tracing for tuberculosis in a low-incidence, high immigration area. Swiss Med Wkly. 2008;138:585-93.

28. Li J, Munsiff SS, Agerton TB. Prevalence of tuberculin skin test positivity in clinical population in New York City. J Immigr Minor Health. 2010;12:816-22.

29. Orlando G, Merli S, Cordier L, et al. Interferon- $\gamma$ releasing assay versus tuberculin skin testing for latent tuberculosis infection in targeted screening programs for high risk immigrants. Infection. 2010;38:195-204.

30. Richards B, Kozak R, Brassard P, Menzies D, Schwartzman K. Tuberculosis surveillance among new immigrants in Montreal. Int J Tuberc Lung Dis. 2005:9:858-64

31. Saracino A, Scotto G, Fornabaio C, et al. QuantiFERON-TB Gold In-Tube test (QFT-GIT) for the screening of latent tuberculosis in recent immigrants to Italy. New Microbiol. 2009;32:369-76.

32. Wells Charles D, Zuber Patrick LF, Nolan Charles M, Binkin Nancy J, Goldberg SV. Tuberculosis Prevention Among Foreign-born Persons in Seattle-King County, Washington. Am J Respir Crit Care Med. 1997;156:573-7.

33. Winje B, Oftung F, Korsvold G, et al. Screening for tuberculosis infection among newly arrived asylum seekers: Comparison of QuantiFERON(R)TB Gold with tuberculin skin test. BMC Infect Dis. 2008;8:65.

34. Lucas M, Nicol P, McKinnon E, et al. A prospective large-scale study of methods for the detection of latent Mycobacterium tuberculosis infection in refugee children. Thorax. 2010;65:442-8.

35. Minodier P, Vr L, Carle M-E, Blais D, Ovetchkine P, Tapiero B. Evaluation of a school-based program for diagnosis and treatment of latent tuberculosis infection in immigrant children. J Infect Public Health. 2010;3:67-75.

36. Fortin K, Carceller A, Robert M, Chevalier I, Lamarre V, Lebel MH. Prevalence of positive tuberculin skin tests in foreign-born children. J Paediatr Child Health. 2007:43:768-72.

37. Méndez-Echevarría A, González-Muñoz M, Mellado MJ, et al. Interferon- $\gamma$ release assay for the diagnosis of tuberculosis in children. Arch Dis Child. 2012;97(6):514-6

38. Bodenmann $\mathrm{P}$, Vaucher $\mathrm{P}$, Wolff $\mathrm{H}$, et al. Screening for latent tuberculosis infection among undocumented immigrants in Swiss healthcare centres; a descriptive exploratory study. BMC Infect Dis. 2009;9:34.

39. Hardy AB, Varma R, Collyns T, Moffitt SJ, Mullarkey C, Watson JP. Costeffectiveness of the NICE guidelines for screening for latent tuberculosis infection: the QuantiFERON-TB Gold IGRA alone is more cost-effective for immigrants from high burden countries. Thorax. 2010;65:178-80.

40. Kik SV, Franken WPJ, Arend SM, et al. Interferon-gamma release assays in immigrant contacts and effect of remote exposure to Mycobacterium tuberculosis. Int J Tuberc Lung Dis. 2009;13:820-8.

41. Pareek M, Watson JP, Ormerod LP, et al. Screening of immigrants in the UK for imported latent tuberculosis: a multicentre cohort study and costeffectiveness analysis. Lancet Infect Dis. 2011;11:435-44.

42. Pareek $M$, Bond $M$, Shorey J, et al. Community-based evaluation of immigrant tuberculosis screening using interferon $\gamma$ release assays and tuberculin skin testing: observational study and economic analysis. Thorax. 2013;68:230-9.

43. Liu Y, Posey DL, Cetron MS, Painter JA. Effect of a Culture-Based Screening Algorithm on Tuberculosis Incidence in Immigrants and Refugees Bound for the United StatesA Population-Based Cross-sectional StudyTB Screening in Immigrants and Refugees Bound for the United States. Ann Intern Med. 2015;162:420-8

44. Campbell J, Krot J, Elwood K, Cook V, Marra F. A Systematic Review on TST and IGRA Tests Used for Diagnosis of LTBI in Immigrants. Mol Diagn Ther. 2015:19:9-24.

45. Campbell J, Chen W, Johnston J, et al. Latent Tuberculosis Infection Screening in Immigrants to Low-Incidence Countries: A Meta-Analysis. Mol Diagn Ther. 2015;19:107-17.
46. Lillebaek T, Andersen AB, Dirksen A, Smith E, Skovgaard LT, Kok-Jensen A. Persistent high incidence of tuberculosis in immigrants in a low-incidence country. Emerg Infect Dis. 2002;8:679-84.

47. MacPherson DW, Gushulak BD. Balancing prevention and screening among international migrants with tuberculosis: population mobility as the major epidemiological influence in low-incidence nations. Public Health. 2006;120:712-23.

48. Marks GB, Bai J, Stewart GJ, Simpson SE, Sullivan EA. Effectiveness of postmigration screening in controlling tuberculosis among refugees: a historical cohort study, 1984-1998. Am J Public Health. 2001;91:1797-9.

49. Choudhury IW, West CR, Ormerod LP. The outcome of a cohort of tuberculinpositive predominantly South Asian new entrants aged 16-34 to the UK: Blackburn 1989-2001. J Public Health. 2014;36:390-5.

50. Harstad I, Winje BA, Heldal E, Oftung F, Jacobsen GW. Predictive values of QuantiFERON-TB Gold testing in screening for tuberculosis disease in asylum seekers [Short communication]. Int J Tuberc Lung Dis. 2010;14:1209-11.

51. Marks GB, Bai J, Simpson SE, Sullivan EA, Stewart GJ. Incidence of tuberculosis among a cohort of tuberculin-positive refugees in Australia: reappraising the estimates of risk. Am J Respir Crit Care Med. 2000;162:1851-4.

52. Jeon CY, Murray MB. diabetes mellitus increases the risk of active tuberculosis: a systematic review of 13 observational studies. PLoS Med. 2008:5, e152.

53. Cobelens FGJ, van Deutekom H, Draayer-Jansen IWE, et al. Risk of infection with Mycobacterium tuberculosis in travellers to areas of high tuberculosis endemicity. Lancet. 2000;356:461-5.

54. McCarthy OR. Asian immigrant tuberculosis-the effect of visiting Asia. Br J Dis Chest. 1984;78:248-53.

55. Kik SV, Mensen M, Beltman M, et al. Risk of travelling to the country of origin for tuberculosis among immigrants living in a low-incidence country. Int J Tuberc Lung Dis. 2011;15:38-43.

56. Maguire H, Dale JW, MCHugh TD, et al. Molecular epidemiology of tuberculosis in London 1995-7 showing low rate of active transmission. Thorax. 2002;57:617-22.

57. Fok A, Numata Y, Schulzer M, FitzGerald MJ. Risk factors for clustering of tuberculosis cases: a systematic review of population-based molecular epidemiology studies [Review Article]. Int J Tuberc Lung Dis. 2008; 12:480-92.

58. Diel R, Rusch-Gerdes S, Niemann S. Molecular epidemiology of tuberculosis among immigrants in Hamburg, Germany. J Clin Microbiol. 2004;42:2952-60.

59. Cohen T, Lipsitch M, Walensky RP, Murray M. Beneficial and perverse effects of isoniazid preventive therapy for latent tuberculosis infection in HIV-tuberculosis coinfected populations. Proc Natl Acad Sci U S A. 2006;103:7042-7.

60. Currie CSWB, Cheng RC, Dye C. Tuberculosis epidemics driven by HIV: is prevention better than cure? AIDS. 2003;17:2501-8.

61. Dye C, Williams BG. Eliminating human tuberculosis in the twenty-first century. J R Soc Interface. 2008;5:653-62.

62. Ziv E, Daley CL, Blower SM. Early therapy for latent tuberculosis infection. Am J Epidemiol. 2001;153:381-5.

63. Murray CJL, Salomon JA. Modeling the impact of global tuberculosis control strategies. Proc Natl Acad Sci U S A. 1998;95:13881-6.

64. World Health Organisation. Guidelines on the management of latent tuberculosis infection. Geneva: World Health Organisation; 2014.

65. Coker R, Bell A, Pitman R, et al. Tuberculosis screening in migrants in selected European countries shows wide disparities. Eur Respir J. 2006;27:801-7.

66. Rieder HL, Zellweger JP, Raviglione MC, Keizer ST, Migliori GB. Tuberculosis control in Europe and international migration. Eur Respir J. 1994:7:1545-53.

67. Alvarez G, Gushulak B, Rumman K, et al. A comparative examination of tuberculosis immigration medical screening programs from selected countries with high immigration and low tuberculosis incidence rates. BMC Infect Dis. 2010;11:3.

68. Alvarez GG, Clark M, Altpeter E, et al. Pediatric tuberculosis immigration screening in high-immigration, low-incidence countries. Int J Tuberc Lung Dis. 2010;14:1530-7.

69. Pareek M, Baussano I, Abubakar I, Dye C, Lalvani A. Evaluation of immigrant tuberculosis screening in industrialized countries. Emerg Infect Dis. 2012;18:1422-9

70. Zenner D, Southern J, van Hest R, et al. Active case finding for tuberculosis among high-risk groups in low-incidence countries. Int J Tuberc Lung Dis. 2013;17:573-82. State of the art series. Case finding/screening. Number 3 in the series. 
71. Aldridge RW, Yates TA, Zenner D, White PJ, Abubakar I, Hayward AC. Preentry screening programmes for tuberculosis in migrants to low-incidence countries: a systematic review and meta-analysis. Lancet Infect Dis. 2014;14:1240-9.

72. Public Health England. UK pre-entry tuberculosis screening report 2014. London: Public Health England; 2014.

73. Public Health England. Tuberculosis in the UK: 2015 report. London: Public Health England; 2015.

74. Denkinger CM, Dheda K, Pai M. Guidelines on interferon- $\gamma$ release assays for tuberculosis infection: concordance, discordance or confusion? Clin Microbiol Infect. 2011;17:806-14.

75. Bettache N, Schwartzman K, Cnossen S, et al. Effectiveness of post-arrival latent tuberculosis screening programs in the foreign born: a systematic review and meta-analysis. In: B58 tuberculosis education and surveillance. San Francisco: American Thoracic Society; 2012. A3325-A

76. Public Health England. Collaborative tuberculosis strategy for England 2014 to 2019. London: Public Health England; 2014.

77. Dasgupta K, Schwartzman K, Marchand R, Tennenbaum TN, Brassard P, Menzies D. Comparison of cost-effectiveness of tuberculosis screening of close contacts and foreign-born populations. Am J Respir Crit Care Med. 2000;162:2079-86.

78. Khan K, Muennig P, Behta M, Zivin JG. Global drug-resistance patterns and the management of latent tuberculosis infection in immigrants to the United States. N Engl J Med. 2002;347:1850-9.

79. Linas BP, Wong AY, Freedberg KA, Horsburgh CR Jr. Priorities for screening and treatment of latent tuberculosis infection in the United States. Am J Respir Crit Care Med. 2011;184:590-601

80. National Collaborating Centre for Chronic Conditions. Tuberculosis: clinical diagnosis and management of tuberculosis, and measures for its prevention and control. London: Royal College of Physicians; 2006.

81. National Collaborating Centre for Chronic Conditions. Tuberculosis: clinical diagnosis and management of tuberculosis, and measures for its prevention and control. London: Royal College of Physicians; 2011.

82. Oxlade $\mathrm{O}$, Schwartzman K, Menzies D. Interferon-gamma release assays and TB screening in high-income countries: a cost-effectiveness analysis. Int J Tuberc Lung Dis. 2007;11:16-26.

83. Schwartzman K, Menzies D. Tuberculosis screening of immigrants to lowprevalence countries. a cost-effectiveness analysis. Am J Respir Crit Care Med. 2000;161:780-9.

84. Iqbal AZ, Leighton J, Anthony J, Knaup RC, Peters EB, Bailey TC. Costeffectiveness of Using Quantiferon Gold (QFT-G) ${ }^{\oplus}$ versus Tuberculin Skin Test (TST) among U.S. and Foreign Born Populations at a Public Health Department Clinic with a Low Prevalence of Tuberculosis. Public Health Nurs. 2014:31:144-52.

85. Porco TC, Lewis B, Marseille E, Grinsdale J, Flood JM, Royce SE. Costeffectiveness of tuberculosis evaluation and treatment of newly-arrived immigrants. BMC Public Health. 2006;6:157.

86. Zammarchi $L$, Casadei $G$, Strohmeyer $M$, et al. A scoping review of costeffectiveness of screening and treatment for latent tuberculosis infection in migrants from high-incidence countries. BMC Health Serv Res. 2015;15:1-11.

87. Lalvani A, Pareek M. A 100 year update on diagnosis of tuberculosis infection. Br Med Bull. 2009;93:69-84.

88. European Centre for Disease Prevention and Control. Evidence-based guidance: prevention of infectious diseases among newly arrived migrants in the EU/EEA. Stockholm: ECDC; 2015.

\section{Submit your next manuscript to BioMed Central and we will help you at every step:}

- We accept pre-submission inquiries

- Our selector tool helps you to find the most relevant journal

- We provide round the clock customer support

- Convenient online submission

- Thorough peer review

- Inclusion in PubMed and all major indexing services

- Maximum visibility for your research

Submit your manuscript at www.biomedcentral.com/submit 